\title{
Long-term effects of high-fat or high-carbohydrate diets on glucose tolerance in mice with heterozygous carnitine palmitoyltransferase-1a deficiency
}

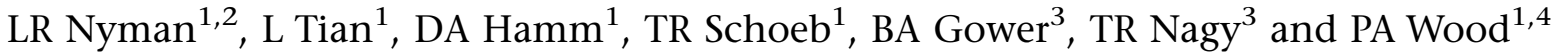 \\ ${ }^{1}$ Department of Genetics, University of Alabama Birmingham, Birmingham, AL, USA; ${ }^{2}$ Division of Diabetes, Endocrinology \\ and Metabolism, Department of Medicine, Vanderbilt University, Nashville, TN, USA; ${ }^{3}$ Department of Nutrition Sciences, \\ University of Alabama Birmingham, Birmingham, AL, USA and ${ }^{4}$ Metabolic Signaling \& Disease Program, Diabetes and \\ Obesity Research Center, Sanford-Burnham Medical Research Institute at Lake Nona, Orlando, FL, USA
}

Background: Abnormal fatty acid metabolism is an important feature in the mechanisms of insulin resistance and $\beta$-cell dysfunction. Carnitine palmitoyltransferase-1 a (CPT-1a, liver isoform) has a pivotal role in the regulation of mitochondrial fatty acid oxidation. We investigated the role of CPT-1a in the development of impaired glucose tolerance using a mouse model for CPT-1 a deficiency when challenged by either a high-carbohydrate (HCD) or a high-fat diet (HFD) for a total duration of up to 46 weeks.

Methods: Insulin sensitivity and glucose tolerance were assessed in heterozygous CPT-1a-deficient (CPT-1a $+/-)$ male mice after being fed either a HCD or a HFD for durations of 28 weeks and 46 weeks. Both glucose and insulin tolerance tests were used to investigate $\beta$-cell function and insulin sensitivity. Differences in islet insulin content and hepatic steatosis were evaluated by morphological analysis.

Results: CPT-1a+/- mice were more insulin-sensitive than CPT-1a+/ + mice when fed either HCD or HFD. The increased insulin sensitivity was associated with an increased expression of Cpt-1b (muscle isoform) in liver, as well as increased microvesicular hepatic steatosis compared with CPT-1a+/+ mice. CPT-1a +/- mice were more glucose tolerant than CPT$1 \mathrm{a}+/+$ mice when fed the HCD, but there was no significant difference when fed HFD. Moreover, CPT- $1 \mathrm{a}+/-$ mice fed HFD or HCD had fewer and smaller pancreatic islets than CPT-1a + / + mice.

Conclusions: CPT-1 a deficiency preserved insulin sensitivity when challenged by long-term feeding of either diet. Furthermore, CPT-1 a-deficient mice had distinct phenotypes dependent on the diet fed demonstrating that both diet and genetics collectively have a role in the development of impaired glucose tolerance.

Nutrition and Diabetes (2011) 1, e14; doi:10.1038/nutd.2011.11; published online 22 August 2011

Keywords: carnitine palmitoyltransterase-1a deficiency; mouse model; glucose tolerance; fatty acid oxidation disorders

\section{Introduction}

Impaired glucose tolerance is considered to be the earliest identifiable metabolic abnormality in the pathogenesis of Type 2 diabetes. ${ }^{1}$ Glucose tolerance is thought to be a function of both $\beta$-cell responsiveness to increased blood glucose concentration and peripheral tissue responsiveness to insulin. ${ }^{2,3}$ Many metabolic phenotypes are influenced

Correspondence: Dr PA Wood, Sanford-Burnham Medical Research Institute at Lake Nona, 6400 Sanger Road, Orlando, FL 32827, USA.

E-mail: pwood@sanfordburnham.org

Received 28 February 2011; revised 20 June 2011; accepted 10 July 2011 by a combination of genotype and environment, particularly the diet.

Aberrant mitochondrial fatty acid oxidation (FAO) may have a pivotal role in the development of impaired glucose tolerance. ${ }^{4-6}$ Carnitine palmitoyltransferase-1 (CPT-1) functions as the rate-limiting step of FAO. This enzyme is responsible for converting long-chain acyl-CoAs into longchain acylcarnitines, a process required for translocation across the mitochondrial membrane into the mitochondrial matrix where $\beta$-oxidation occurs. A build-up of fatty acid metabolites, including long-chain fatty acyl-CoAs and diacylglycerols in the cytosol of skeletal muscle and other tissues has been implicated in fatty acid-induced insulin resistance. ${ }^{4}$ Moreover, studies in rodents have shown that 
chronic exposure to fatty acids alters $\beta$-cell function. ${ }^{7-12}$ We have previously demonstrated a range of metabolic phenotypes in mice with inherited deficiencies of other mitochondrial FAO enzymes. ${ }^{13,14}$

Our aim was to systematically investigate factors thought to be involved in the development of insulin resistance and Type 2 diabetes by challenging both CPT- $1 \mathrm{a}+/+$ or CPT-1a + / - mice with a high-carbohydrate diet (HCD) or a high-fat diet (HFD) diet and evaluate them at an age more similar to human middle age. The rationale behind the HCD challenge was to induce conditions that would promote high levels of malonyl-CoA, a potent CPT-1 inhibitor. ${ }^{15}$ Our aim in using the HFD was to challenge this impaired CPT-1 system with excess substrate to promote build-up of fatty acids. We hypothesized that both the HCD-induced increase in the CPT-1a inhibitor, malonyl-CoA, as well as the HFD-induced substrate overload, would unmask a phenotype in a mouse model with a genetically compromised state of FAO. Furthermore, we expected to demonstrate that the combination of chronic environmental stresses of diet and the genetic limitation of FAO by decreased CPT-1a activity would lead to one or all of the metabolic abnormalities associated with the development of Type 2 diabetes.

\section{Materials and methods}

\section{Animals}

Male mice with a heterozygous deficiency in CPT-1a (CPT$1 \mathrm{a}+/$ - mice) (129S6/SvEvTac-coisogenic background) were previously described. ${ }^{16}$ Homozygous CPT-1a-deficient mice are not viable. ${ }^{16}$ Two separate, age-matched (CPT-1a $+/+$ and CPT-1a + /-) groups of mice were maintained on either a HFD or HCD for either 28 or 46 weeks. All mice were housed in a pathogen-free facility (12-h light/12-h dark cycle). All experiments were approved by the Institutional Animal Care and Use Committee of the University of Alabama at Birmingham.

\section{Diets, body weight and body composition analyses}

Mice were fed either a HFD (Teklad diet-TD 97268) containing $43 \%$ fat (calorie \%) using beef tallow as the major fat source, or a HCD (Teklad diet-TD 97267) that was 14\% fat, with starch making up the bulk of calories. Both diets were isocaloric and contained equal amounts of sucrose. Mice (17- to 19-day-olds) were weaned onto their respective diets and fed ad libitum. Body weights were determined weekly using a standard balance. Body composition was determined at the end of the study period using dual-energy X-ray absorptometry, as described previously. ${ }^{17,18}$

\section{Serum metabolite assays}

Mice were fasted for $20-22 \mathrm{~h}$ before collection of serum. Total non-esterified fatty acids were measured by an enzymatic, colorimetric method ('NEFA-C' reagents, Wako Diagnostics, Richmond, VA, USA). Triglyceride concentrations were measured in $10 \mu \mathrm{l}$ sera with the Ektachem DT II system (Johnson and Johnson Clinical Diagnostics, Rochester, NY, USA).

\section{Glucose tolerance tests}

The oral glucose tolerance test outcomes of interest included basal (fasted) serum glucose concentration, glucose and insulin concentrations at individual time points following glucose ingestion. From these data, the rate of decline of blood glucose was determined.

At $20 \mathrm{~h}$ before performing oral glucose tolerance test, animals were food-deprived with water overnight. After the mice were weighed, a baseline blood sample was obtained by tail bleed, and additional blood samples were obtained at 15, 30,60 and 120 min following oral glucose administration at $2.0 \mathrm{mg}$ per g body weight.

Insulinogenic index calculations were made to assess differences in first-phase insulin secretion. The formula used is ((insulin) $30 \mathrm{~min}-($ insulin) $0 \mathrm{~min}) /($ glucose $) 30 \mathrm{~min}-$ (glucose) $0 \mathrm{~min}) .{ }^{19}$ This represents the change in insulin level at $30 \mathrm{~min}$ after glucose administration divided by the change in glucose level $30 \mathrm{~min}$ after the oral dose of glucose.

\section{Insulin tolerance tests}

Mice were deprived of food for $4 \mathrm{~h}$, baseline blood samples were collected and recombinant human insulin (Humulin R, $100 \mathrm{U} \mathrm{ml}^{-1}$ stock) was injected intraperitoneally $(0.1 \mathrm{ml}$ per $10 \mathrm{~g}$ body weight). Before injection, insulin was diluted in saline to a concentration of $0.075 \mathrm{U} \mathrm{ml}^{-1}$ (1:1330). Additional blood samples were obtained at 10, 20 and $30 \mathrm{~min}$, following insulin injection. Outcomes of interest included the final $(30 \mathrm{~min})$ glucose concentration, and the rate of decline of glucose, which was calculated from the slope of the descending points after insulin injection. The rate of decline of blood glucose was determined to be a reflection of the degree of insulin sensitivity.

Blood glucose concentrations were measured with a blood glucose meter (Accu-chek; Roche Diagnostics, Indianapolis, IN, USA), and serum insulin levels were measured with a rat sensitive immunoassay kit (LINCO Research, St Charles, MO, USA) with a 1:10 dilution of mouse serum used as per the manufacturer's instructions.

\section{RNA extraction and real-time quantitative RT-PCR analysis} Total RNA was extracted from heart, liver and skeletal muscle (soleus and gastrocnemius) using TRIzol (Invitrogen Life Technologies, Carlsbad, CA, USA). First-strand cDNA was generated from $5 \mu \mathrm{g}$ of RNA in a $20 \mu \mathrm{l}$ volume reaction containing both oligo-(dT) and random hexamers (Invitrogen First-Strand Synthesis Kit). Real-time quantitative RT-PCR (QRT-PCR) was carried out in a $20 \mu$ l reaction volume containing $10 \mu \mathrm{l}$ supermix UDG (Invitrogen Life 
Technologies Inc.), $2 \mu \mathrm{l}$ cDNA, $0.2 \mu \mathrm{M}$ LUX Cpt-1a and 1b, primer forward, $0.2 \mu \mathrm{M}$ LUX $C p t-1 a$ and $1 b$, primer reverse (FAM-labeled, Invitrogen Life Technologies), $0.1 \mu \mathrm{M}$ 18S RNA primer forward, $0.1 \mu \mathrm{M} 18 \mathrm{~S}$ RNA primer reverse (JOE-labeled, Invitrogen, Life Technologies). Cycling conditions included incubation at $50^{\circ} \mathrm{C}$ for $2 \mathrm{~min}$, a $2 \mathrm{~min} 95^{\circ} \mathrm{C}$ denaturing step, followed by 44 cycles of $95^{\circ} \mathrm{C}$ denaturation, $45 \mathrm{~s}$ annealing time at $58^{\circ} \mathrm{C}$ and a $30 \mathrm{~s}$ extension step at $72^{\circ} \mathrm{C}$. A melting curve analysis was completed following amplification from $55-95{ }^{\circ} \mathrm{C}$ to assure product identification and homogeneity.

Each sample was normalized to the housekeeping gene actin to compensate for any differences in starting quantity of total RNA. The relative quantification for $C p t-1 b$ and Cpt-1a mRNA in CPT-1a +/- compared with CPT-1a $+/+$ was calculated using the $\Delta \Delta C_{\mathrm{t}}=2 \exp -\left(\Delta C_{\mathrm{t}}\right.$ sample $(\mathrm{CPT}-1 \mathrm{a}+/-)-\Delta C_{\mathrm{t}}$ reference $\left.(\mathrm{CPT}-1 \mathrm{a}+/+)\right)^{20}$ where $C_{\mathrm{t}}$ is the cycle number when threshold fluorescence is significantly above background. The fold-change is then established by comparing CPT- $1 \mathrm{a}+/-$ sample (relative quantification $=$ calculated $\Delta \Delta C_{\mathrm{t}}$ ) against CPT- $1 \mathrm{a}+/+$ reference (relative quantification $=1$ ).

\section{Histology and morphometric analyses of liver and pancreas}

Liver and pancreas were collected from mice fasted for $24 \mathrm{~h}$ who had been fed a HCD or HFD for 46 weeks, processed routinely for paraffin sectioning and stained with hematoxylin and eosin. Hepatocellular fatty change was assessed by subjective scoring. The evaluator examined the slides without knowledge of genotype or diet classification. Microvesicular and macrovesicular fatty changes, each were scored 0 for normal/absent, 1 for mild, 2 for moderate and 3 for severe, using increments of 0.5 for intermediate degrees. Estimated distribution of each type of change was scored 1 for up to $1 / 4$ of the section, 2 for $1 / 4$ to $1 / 2,3$ for $1 / 2$ to $3 / 4$ or 4 for $3 / 4$ to all of the section. Severity scores for microvesicular and macrovesicular fatty changes were determined by multiplying the scores of severity and distribution and were summed for a total severity score. Size and numbers of islets in immunohistochemically stained sections of pancreas were determined by histomorphometry. Images of each section were made at $\times 1$ with a Nikon Super CoolScan 5000ED scanner (Melville, NY, USA) at 4000 pixels per inch. For each section, the total area of pancreas and the area of each immunostained islet were determined with Image-Pro Plus 5.1 image analysis software (Media Cybernetics, Silver Spring, MD, USA), and average islet area in $\mathrm{mm}^{2}$, number of islets per $\mathrm{mm}^{2}$ pancreas, and the ratio of total islet area to pancreas area were calculated.

\section{Immunohistochemistry}

Paraffin sections of pancreas were stained for insulin according to a modification of a previously reported method. ${ }^{21}$ Briefly, endogenous peroxides were blocked with $0.3 \%$ hydrogen peroxide in phosphate-buffered saline for $30 \mathrm{~min}$. Background staining was decreased by blocking with
$1 \%$ bovine serum albumin in phosphate-buffered saline for $1 \mathrm{~h}$. Anti-pig insulin (Sigma-Aldrich, St Louis, MO, USA) developed in guinea pig, was used as the primary antibody at 1:9000 dilution and incubated for $2 \mathrm{~h}$ in a humidity chamber at room temperature. Anti-guinea pig IgG (whole molecule) peroxidase conjugate (Sigma-Aldrich) at 1:1000 dilution was used for the secondary antibody and incubated for $30 \mathrm{~min}$ at room temperature in a humidity chamber. True blue peroxidase substrate (Kierkegaard and Perry Laboratories, Gaithersburg, MD, USA) was applied for $10 \mathrm{~min}$ and washed in water for $3 \mathrm{~min}$. Sections were counterstained with Nuclear Fast Red (Vector Labs, Burlingame, CA, USA) for 10 min followed with a 3 min wash in water, then air-dried and coverslipped with Permount (Fisher Chemicals, Fairlawn, NJ, USA).

\section{Statistics}

All data were expressed as mean values with s.e.m. Student's $t$-tests and two-way analysis of variance were used to analyze effects of genotype and diet. Liver macrovesicular and microvesicular fat scores were analyzed using the KruskalWallis test, the rank-sum test with Tukey's probability correction for multiple comparisons, and analysis of variance of ranks. $P$-values $<0.05$ were considered significant.

\section{Results}

Clinical markers for insulin resistance, impaired glucose tolerance and Type 2 diabetes

We evaluated clinical markers of obesity, insulin resistance and Type 2 diabetes such as body weight, body fat and liver fatty change in CPT-1a $+/-$ mice as compared with the CPT-1a $+/+$ mice fed either HCD or HFD.

Using dual-energy X-ray absorptometry analysis, body fat was significantly greater in CPT-1a $+/-$ mice fed HCD for 46 weeks than for CPT-1a $+/+$ mice $(P=0.0241)$ (Figure $1 \mathrm{a})$, although there were no significant differences in body weight of CPT-1a+/- mice (Figure 1c) as compared with CPT- $1 \mathrm{a}+/+$ mice.

In contrast to the findings on the HCD, body fat was significantly greater in CPT-1a $+/+$ mice fed HFD for 46 weeks compared with CPT- $1 \mathrm{a}+/-$ mutants $(P=0.00019)$ (Figure $1 \mathrm{~b}$ ). The body weights of the oldest CPT-1a $+/-$ mice were significantly lower than CPT- $1 \mathrm{a}+/+$ mice eating HFD (Figure 1d).

Fasting serum glucose, insulin, triglycerides and free fatty acids were evaluated in mice challenged with either a HCD or HFD for either 28 or 46 weeks (Table 1). CPT-1a + /mice fed HFD had significantly higher serum glucose values at 28 weeks; however, there were no other significant differences in fasting glucose values as compared with CPT-1a $+/+$ mice fed either diet. Fasting insulin levels were significantly lower (28 weeks $P=0.01 ; 46$ weeks $P=0.03)$ in HFD-fed CPT- $1 \mathrm{a}+/-$ mice as compared with 

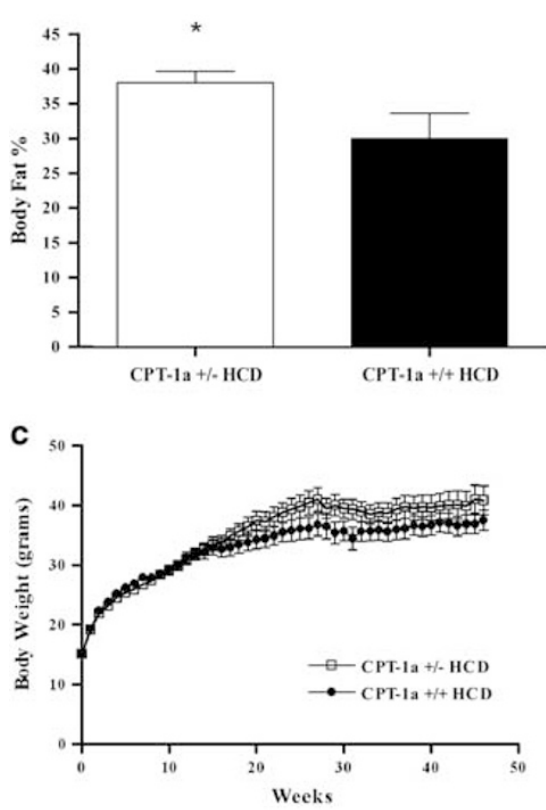

b

High Fat


Figure 1 Effect of diet and CPT-1 a deficiency on body weight and body-fat percentage over 46 weeks. (a) Body fat percentages in CPT-1a $+/-$ mice $(n=17)$ fed a HCD as compared with CPT- $1 \mathrm{a}+/+$ mice $(n=16)$. (b) Body fat percentages in CPT- $1 \mathrm{a}+/-$ mice fed a HFD $(n=16)$ as compared with CPT- $1 \mathrm{a}+/+$ mice $(n=19)$. (c) Body weights for CPT-1a $+/-(n=19)$ versus CPT1 $\mathrm{a}+/+(n=17)$ mice fed HCD for 46 weeks. (d) Body weights for CPT-1a $+/-(n=18)$ versus CPT1 $\mathrm{a}+/+$ $(n=20)$ mice fed HFD for 46 weeks reached significant differences during the final 8 weeks $\left({ }^{\star} P<0.05,{ }^{* *} P<0.01\right)$.

Table 1 Serum metabolites and insulin concentrations

\begin{tabular}{|c|c|c|c|c|c|c|c|}
\hline & & Fasted CPT-1a+/- & Fed CPT-1a+/- & & Fasted CPT-1a+/+ & Fed CPT-1a+/+ & \\
\hline \multicolumn{8}{|l|}{ Serum glucose $\mathrm{mmoll}^{-1}$} \\
\hline HFD, 28 weeks & $n=8$ & $3.66 \pm 0.54^{*}$ & & $n=10$ & $3.00 \pm 0.61^{*}$ & & $P=0.03$ \\
\hline HFD, 46 weeks & $n=9$ & $3.27 \pm 1.3$ & & $n=9$ & $3.11 \pm 0.83$ & & \\
\hline HCD, 28 weeks & $n=9$ & $3.22 \pm 0.88$ & & $n=10$ & $3.83 \pm 2.72$ & & \\
\hline HCD, 46 weeks & $n=9$ & $2.83 \pm 0.67$ & & $n=10$ & $2.78 \pm 0.61$ & & \\
\hline \multicolumn{8}{|l|}{ Serum insulin pmol/ ${ }^{-1}$} \\
\hline HFD, 28 weeks & $n=13$ & $36.1 \pm 10^{*}$ & & $n=11$ & $63.7 \pm 33^{*}$ & & $P=0.01$ \\
\hline HFD, 46 weeks & $n=9$ & $32.7 \pm 13^{*}$ & & $n=8$ & $63.7 \pm 36^{*}$ & & $P=0.03$ \\
\hline HCD, 28 weeks & $n=10$ & $27.5 \pm 14$ & & $n=10$ & $75.7 \pm 75$ & & \\
\hline $\mathrm{HCD}, 46$ weeks & $n=9$ & $27.5 \pm 1.7$ & & $n=9$ & $36.1 \pm 15$ & & \\
\hline \multicolumn{8}{|l|}{ Triglycerides mmoll ${ }^{-1}$} \\
\hline SRD, 24-32 weeks & $n=7$ & $1.59 \pm 0.73$ & $1.19 \pm 0.30^{*}$ & $n=7$ & $1.56 \pm 0.41$ & $1.90 \pm 0.55^{*}$ & $P=0.01$ \\
\hline HFD, 46 weeks & $n=10$ & $1.65 \pm 0.32$ & & $n=13$ & $1.58 \pm 0.43$ & & \\
\hline HCD, 46 weeks & $n=12$ & $1.76 \pm 0.63$ & & $n=11$ & $1.63 \pm 0.25$ & & \\
\hline \multicolumn{8}{|c|}{ Serum free fatty acids mmol ${ }^{-1}$} \\
\hline SRD, 24-32 weeks & $n=7$ & $1.6 \pm 0.38$ & $0.90 \pm 0.2^{*}$ & $n=7$ & $1.6 \pm 0.30$ & $1.3 \pm 0.20^{*}$ & $P=0.018$ \\
\hline HFD, 46 weeks & $n=10$ & $0.82 \pm 0.23$ & & $n=13$ & $0.98 \pm 0.24$ & & \\
\hline HCD, 46 weeks & $n=12$ & $1.2 \pm 0.040$ & & $n=11$ & $0.91 \pm 0.24$ & & \\
\hline
\end{tabular}

Abbreviations: CPT, carnitine palmitoyltransferase; HCD, high-carbohydrate diet; HFD, high-fat diet; SRD, standard rodent diet. Fasting glucose and insulin for CPT- $1 \mathrm{a}+/-$ and CPT $-1 \mathrm{a}+/+$ fed HFD or HCD for 28 weeks or 46 weeks. Fasting and fed (SRD only) free fatty acids and triglycerides for mice fed SRD for 24-32 weeks, HFD and HCD for 28 or 46 weeks.

Figure 2 Effects of diet on glucose and insulin tolerance in CPT-1 $a+/-$ mice after 28 weeks. (a-c) Glucose levels ( $\left.{ }^{* *} P<0.01,{ }^{* \star *} P<0.001\right)$, insulin levels and insulinogenic indexes $\left({ }^{* *} P=0.0038\right)$ throughout glucose tolerance test in CPT- $1 \mathrm{a}+/-(n=10)$ versus CPT-1a $+/+(n=10)$ mice fed HCD. (d) Insulin tolerance test. Slopes for the rate of decline of glucose $\left({ }^{* *} \mathrm{P}<0.002\right)$ in response to an intraperitoneal injection of insulin for CPT- $1 \mathrm{a}+/-(n=8)$ and CPT- $1 \mathrm{a}+/+(n=8)$ mice fed HCD. (e-g) Glucose levels, insulin levels and insulinogenic indexes $\left({ }^{*} P=0.026\right)$ throughout glucose tolerance test in CPT-1a $+/-(n=8)$ versus CPT- $1 \mathrm{a}+/+$ $(n=11)$ mice fed HFD. (h) Insulin tolerance test. Slopes for the rate of decline of glucose $\left({ }^{\star} P<0.0439\right)$ in response to an intraperitoneal injection of insulin for CPT- $1 \mathrm{a}+/-(n=8)$ and CPT- $1 \mathrm{a}+/+(n=8)$ mice fed HFD. 
28 weeks
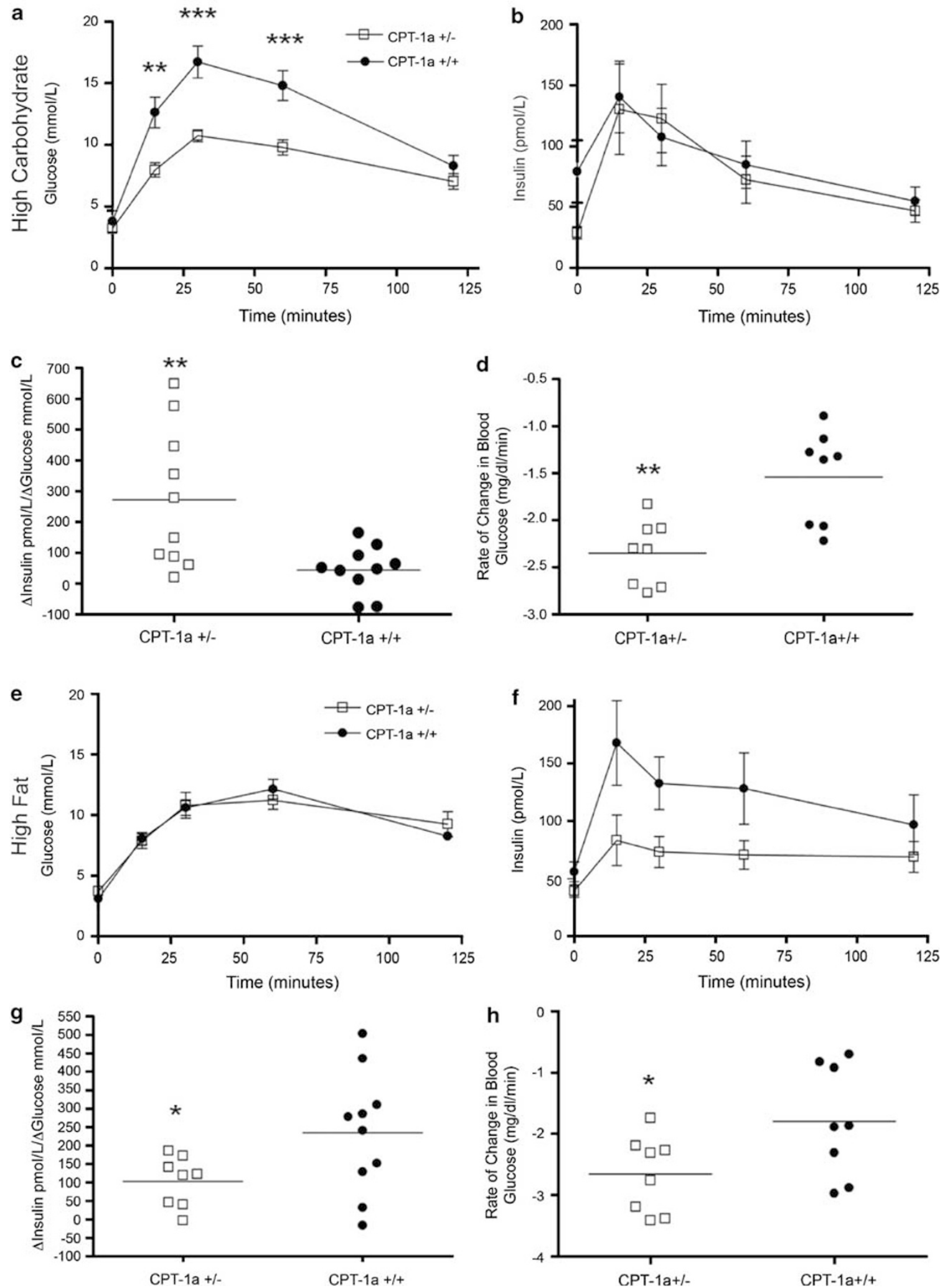


\section{6 weeks}
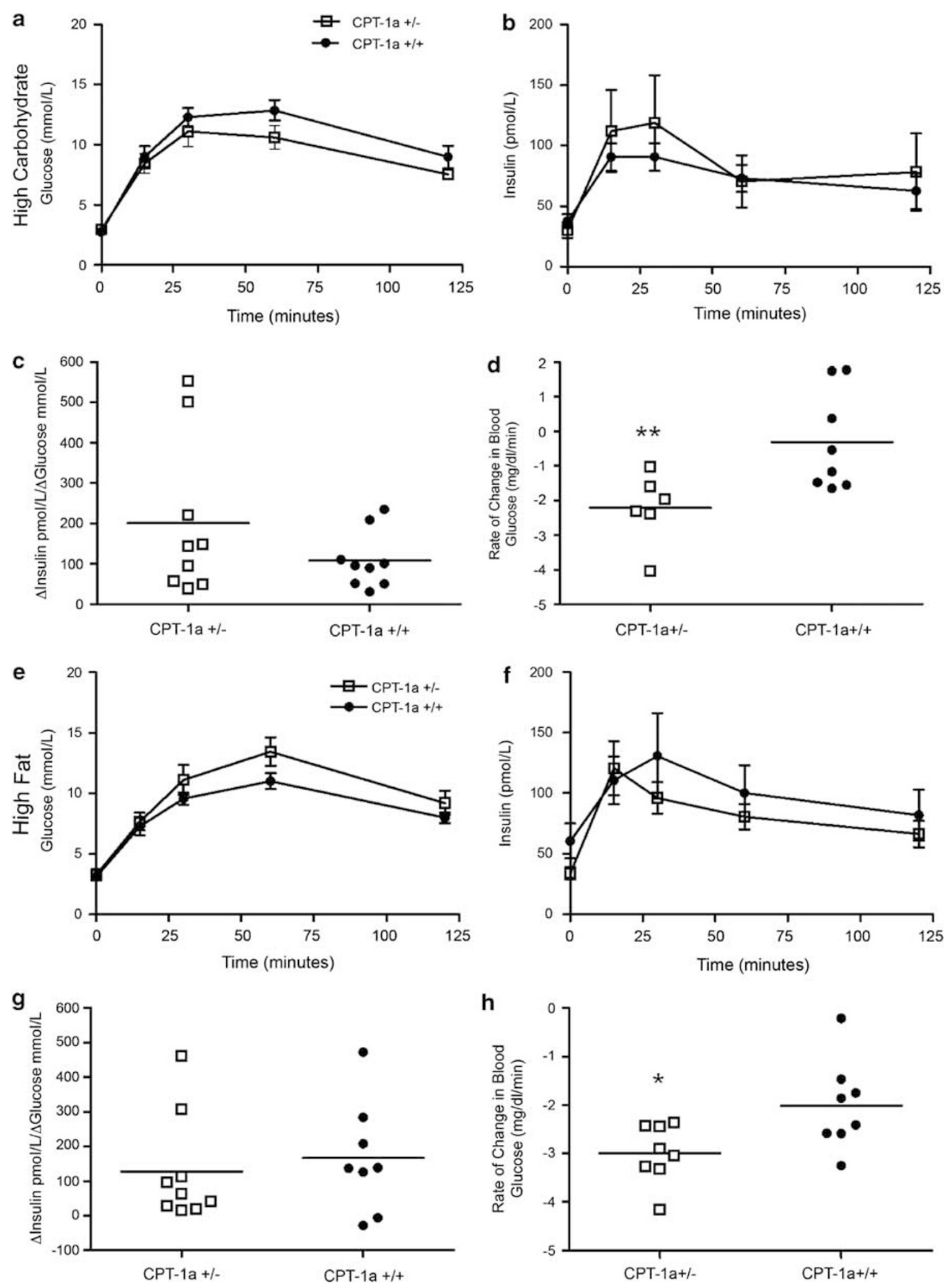
CPT-1a $+/+$ mice, suggesting a state of increased insulin sensitivity. In contrast, there were no significant differences in fasting insulin values among either group fed the HCD. There were no significant differences in fasting serum triglycerides or free fatty acids between CPT- $1 \mathrm{a}+/+$ and $+/-$ mice fed either diet.

\section{Glucose tolerance}

The 28-weeks-HCD group of CPT-1a $+1-$ mice were more glucose tolerant than CPT- $1 \mathrm{a}+/+$ mice, as measured by oral glucose tolerance test ( 28 weeks, HCD); however, these marked differences were lost by 46 weeks (Figures $2 \mathrm{a}$ and $3 \mathrm{a}$ ). Insulin levels, measured throughout oral glucose tolerance tests, were not significantly different between genotypes (Figures $2 \mathrm{~b}$ and $3 \mathrm{~b}$ ). However, in the 28-weeks groups, CPT-1a + / - mice had significantly higher average first-phase insulin responses to a glucose load, based on insulinogenic index values (Figure 2c), indicating heightened $\beta$-cell responsiveness (28-weeks-HCD $P=0.0038$ ). In addition, in the 46-weeks diet-duration groups there was a trend toward higher average first-phase insulin responses to a glucose load (Figure 3c), however, this difference did not reach significance.

In the 28- and 46-weeks-HFD group, there was no significant difference in glucose tolerance between CPT$1 \mathrm{a}+/-$ and CPT-1a $+/+$ mice (Figures $2 \mathrm{e}$ and 3e). In addition, first-phase insulin secretion, expressed by the insulinogenic index, was significantly lower in CPT-1a $+1-$ mice $(P=0.0256)$ fed the HFD for 28 weeks (Figure $2 \mathrm{~g}$ ). However, first-phase insulin secretion in the longer HFD duration was not significantly different (Figure 3f) between CPT- $1 \mathrm{a}+/-$ and CPT- $1 \mathrm{a}+/+$ mice.

In summary, when fed the HCD for 28 weeks, the CPT-1a $+/-$ mice were more glucose tolerant. Moreover, these mice showed higher estimated first-phase insulin secretion, which may partially explain their improved glucose tolerance. In contrast, CPT- $1 \mathrm{a}+/-$ mice fed the HFD were no less glucose tolerant than CPT-1a $+/+$ mice.

\section{Insulin tolerance}

HCD-fed CPT-1a + / - mice were significantly more insulinsensitive (28-weeks-HCD $P<0.002, \quad$ 46-weeks-HCD $P<0.0088$ ) (Figures $2 \mathrm{~d}$ and $3 \mathrm{~d}$ ) than were CPT-1a $+/+$ controls as was found by their steeper rate of decline of blood glucose levels in response to insulin. In fact, the standard protocol had to be modified because the CPT- $1 \mathrm{a}+1-$ mice became hypoglycemic. In addition, HFD-fed CPT-1a + /- mice were significantly more insulin-sensitive (28-weeksHFD $P=0.0439$ and 46-weeks-HFD $P=0.0129$ ) (Figures $2 \mathrm{~h}$ and $3 \mathrm{~h}$ ) than CPT-1a $+/+$ controls as shown by their steeper rate of decline (negative slope) of blood glucose levels in response to insulin.

\section{Morphometric analysis of $\beta$-cells within islets}

CPT-1a + / - mice fed HCD for 46 weeks had lower percent islet area/pancreas area $(P \leqslant 0.001)$, lower total islet area $(P \leqslant 0.001)$ and lower average islet area $(P \leqslant 0.001)$ (Figures $4 \mathrm{a}-\mathrm{c}$ and $\mathrm{g}$ ). CPT-1a + / - mice fed HFD for 46 weeks, also had lower percent islet area/pancreas area $(P=0.006)$, lower total islet area $(P<0.005)$ and lower total islet number $(P=0.001)$ (Figures $4 \mathrm{~d}-\mathrm{f}$ and $\mathrm{h})$. In summary, CPT- $1 \mathrm{a}+/-$ mice fed either of the diet had significantly fewer insulin positive cells. The only differences between the two diets were that the CPT-1a+/mice fed HCD had lower average islet area, indicating that their islets were smaller overall, whereas HFD-fed CPT-1a + /mice had a lower number of islets overall.

\section{Hepatic steatosis}

CPT-1a + / - mice fed either of the challenge diets demonstrated significantly higher total (macro plus microvesicular combined) fatty change $(P=0.01)$ scores than CPT- $1 \mathrm{a}+/+$ controls (Table 2 and Figures $5 \mathrm{a}$ and c). Microvesicular fat scores in mice fed either of the diets were significantly higher than macrovesicular fat scores, independent of genotype $(P=0.003)$ (Table 2). Microvesicular fat scores were significantly higher in CPT-1a $+/-$ mice when fed either diet as compared with CPT- $1 \mathrm{a}+/+$ mice $(P=0.03)$ (Figures $5 \mathrm{a}-\mathrm{d}$, Table 2). Microvesicular liver steatosis is common in $\beta$-oxidation disorders.

\section{Analysis of steady state mRNA levels by northern blot} Northern blot results showed a striking $C p t-1 b$ mRNA expression in liver of CPT- $1 \mathrm{a}+/-$ mice as compared with CPT-1a $+/+$ mice fed a HCD (data not shown). Cpt-1a expression was lower in CPT-1a $+/-$ mice as was expected because of the targeted mutation (data not shown). To further evaluate $C p t-1 a$ and $b$ mRNA expression, we used the more quantitative and sensitive method of quantitative realtime PCR (QRT-PCR).

QRT-PCR analysis of steady-state mRNA levels CPT-1a+/- mice fed HCD showed significantly higher $C p t-1 b$ expression in liver as measured by QRT-PCR than

Figure 3 Effects of diet on glucose and insulin tolerance in CPT-1a $+/-$ mice after 46 weeks. (a-c) Glucose levels, insulin levels and insulinogenic indexes throughout glucose tolerance test in CPT- $1 \mathrm{a}+/-(n=11)$ versus CPT-1a $+/+(n=10)$ mice fed HCD. (d) Insulin tolerance test. Slopes for the rate of decline of glucose $(* \star P=0.0088)$ in response to an intraperitoneal injection of insulin for CPT-1a $+/-(n=6)$ and CPT-1a $+/+(n=9)$ mice fed HCD. (e-g) Glucose levels, insulin levels and insulinogenic indexes throughout glucose tolerance test in male CPT-1a $+/-(n=9)$ versus CPT-1a $+/+(n=9)$ mice fed HFD. (h) Insulin tolerance test. Slopes for the rate of decline of glucose $\left({ }^{*} P=0.0129\right)$ in response to an intraperitoneal injection of insulin for CPT-1a $+/-(n=8)$ and CPT- $1 \mathrm{a}+/+(n=8)$ mice fed HFD. 
a
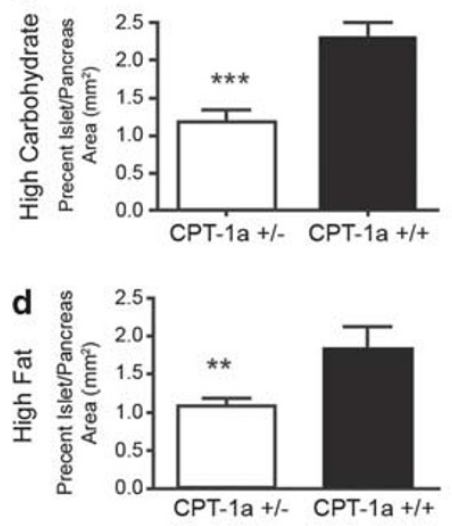

b
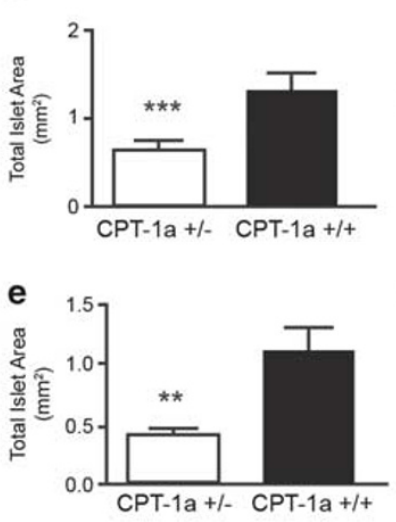

C
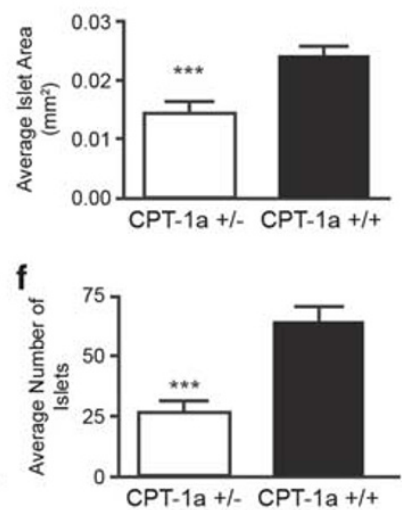
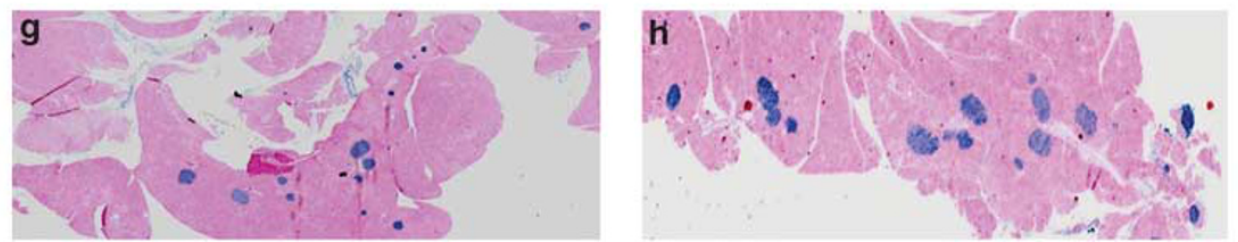

Figure 4 Islet morphology in mice fed a HCD versus a HFD. Differences in (a) \% islet to pancreas area ( $\left.{ }^{\star \star *} P \leqslant 0.001\right)$, (b) total islet area (*** $\left.P \leqslant 0.001\right)$ and mean islet area $(\mathbf{c})\left({ }^{* *} P \leqslant 0.001\right)$ in CPT-1a $+/-(n=7)$ versus CPT-1a $+/+(n=9)$ mice fed a HCD. There were no significant differences in average islet number (data not shown). Differences in (d) \% islet to pancreas area ( $\left.{ }^{*} P=0.006\right),(\mathbf{e})$ total islet area $\left({ }^{\star *} P<0.005\right)$ and $(\mathbf{f})$ average islet number $(* \star \star P=0.001)$ in mice fed a HFD. There were no significant differences in mean islet area (data not shown). CPT-1a $+/-$ mice $(n=10)$ and CPT-1a $+/+$ mice $(n=7)$. Representative images of mouse islet number and size in CPT $1 \mathrm{a}+/-(\mathbf{g})$ and CPT-1a $+/+(\mathbf{h})$ mouse pancreas sections from mice fed the HCD.

Table 2 Liver steatosis in mice fed high-carbohydrate diet or high fat diet for 46 weeks

\begin{tabular}{lcrr}
\hline & $C P T-1 a+/+(H C D)$ & $C P T-1 a+/-(H C D)$ & $C P T-1 a+/+(H F D)$ \\
\hline Macrovesicular fat & $3.5 \pm 3.9$ & $4.7 \pm 3.3$ & $2 \pm 2.2$ \\
Microvesicular fat & $2.4 \pm 4.2$ & $10.1 \pm 10^{*}$ & $2.3 \pm 3.6$ \\
Total & $5.9 \pm 7.6$ & $14.8 \pm 9.6^{*}$ & $6.5 \pm 7.9^{*}$ \\
\hline
\end{tabular}

Abbreviations: CPT, carnitine palmitoyltransferase; HCD, high-carbohydrate diet; HFD, high-fat diet. CPT-1a+/- $(n=22), \mathrm{CPT}-1 \mathrm{a}+/+(n=21)$ mice fed either diet demonstrated significantly higher total (macro plus microvesicular combined) fatty change $(P=0.01)$ as compared with CPT-1a+/+ controls. Microvesicular fat was significantly higher in CPT-1a+/- mice when fed either diet as compared with CPT-1a+/+ mice $(P=0.03)$. Microvesicular fat was significantly greater than macrovesicular fat in mice fed the HCD, independent of genotype $(P=0.003)$.

CPT- $1 \mathrm{a}+/+$ controls $(P<0.05)$. (Figure 5c). HFD-fed CPT-1a $+/-$ mice showed a trend toward higher $C p t-1 b$ expression in liver as measured by QRT-PCR than CPT-1a $+/+$ controls, although the differences were not significant (Figure 5f).

In conclusion of the results section, Table 3 summarizes and provides a contrast of the overall results reported here.

\section{Discussion}

We hypothesized that the long-term effects of a high-calorie diet coupled with a deficiency in mitochondrial FAO (CPT-1a deficiency) would lead to both insulin resistance and $\beta$-cell dysfunction, pivotal features in development of Type 2 diabetes. This initial hypothesis was based on previous studies using CPT-1 inhibitors, ${ }^{5,6,12}$ results from earlier studies in our group showing the effects of another FAO deficiency, long-chain acyl-CoA dehydrogenase, ${ }^{13}$ and other studies associating excess fatty acids with development of insulin resistance and $\beta$-cell dysfunction. ${ }^{4,7,10}$

We metabolically challenged the CPT- $1 \mathrm{a}+/-$ mice with diets either high in fat (HFD) or high in carbohydrates (HCD) to promote for conditions favorable to the generation of malonyl-CoA, a potent inhibitor of CPT-1 activity. Post transcriptionally, CPT-1b (muscle isoform) is regulated solely by changes in malonyl-CoA levels, whereas CPT-1a, the hepatic isoform (CPT-1a) is regulated by both concentrations of and changes in sensitivity to malonyl-CoA. ${ }^{15,22}$ Thus, CPT-1a is unique in that it responds to physiological states such as starvation, insulin deficiency and hypo- and hyperthyroidism by changing its sensitivity to malonyl CoA. $^{22}$ 



Figure 5 Liver steatosis and Cpt-1 b expression in CPT-1 $\mathrm{a}+/-$ and CPT-1 $\mathrm{a}+/+$ mice. Representative images of micro- and macrovesicular fat in liver from (a) CPT $1 \mathrm{a}+/-$ mouse and (b) CPT-1 $\mathrm{a}+/+$ mice fed HCD. Representative images of micro- and macro vesicular fat in liver from (d) CPT $1 \mathrm{a}+/-$ and $(\mathbf{e})$ CPT-1 $\mathrm{a}+/+$ mice fed HFD. Quantitative real-time PCR analysis of Cpt-1b expression in liver as measured by QRT-PCR in (c) HFC-fed CPT-1a $+/-$ mice as compared with controls $(P<0.05)$ and $(f)$ HFD-fed mice.

Table 3 Overall summary of results contrasting the CPT-1a+/- mice and their CPT-1a+/+ controls fed either the high carbohydrate or high fat diets

\begin{tabular}{lll}
\hline & High-carbohydrate diet CPT1 a+/- versus CPT-1a+/+ mice & High-fat diet CPT1a+/- versus CPT-1a+/+ mice \\
\hline $\begin{array}{l}\text { Glucose tolerance } \\
\text { Insulin secretion }\end{array}$ & More glucose tolerant & No significant difference \\
& Higher first-phase insulin secretion & Lower first phase at 28 weeks \\
Insulin tolerance & More insulin-sensitive & No significant difference at 46 weeks \\
Hepatic steatosis & Increased microvesicular fat & More insulin-sensitive \\
Aberrant hepatic CPT-1b expression & Increased & No significant difference, trend is elevated \\
Pancreatic islet morphology & Smaller islets & Fewer islets \\
Body weight & No significant difference & Slightly lower \\
Body fat & Higher & Lower \\
Fasting insulin & No significant difference & Lower \\
\hline
\end{tabular}

Abbreviation: CPT, carnitine palmitoyltransferase

Evidence supporting our initial hypothesis included a study showing that rats fed a high-fat or low-fat diet with etomoxir (a CPT-1 inhibitor) were significantly less insulinsensitive than untreated rats. ${ }^{5}$ However, our most striking and consistent finding was that the CPT-1a $+/-$ mice were more insulin-sensitive than CPT- $1 \mathrm{a}+/+$ mice, when fed either a HCD or HFD, as shown by both insulin tolerance and fasting insulin levels. These results are contrary to our initial hypothesis. We considered the possibility that our results could be explained by the increased $C p t-1 b$ expression in the liver in CPT-1a $+/-$ mice. This mechanism may be similar to that described for overexpression of $C p t-1 b$ in muscle, resulting in improved insulin sensitivity in mice challenged with a HFD. ${ }^{23}$ We speculate that CPT-1b expressed in liver is out of its normal malonyl-CoA regulatory milieu and would be constitutively more active than the usual CPT-1a. This is likely because, CPT-1a is more sensitive to hormonal changes, which increase or decrease its sensitivity to malonyl-CoA in liver, unlike Cpt1b, which is regulated solely by changes in the concentrations of malonyl-CoA. The increased insulin sensitivity in the CPT-1 + /- mice, especially given the HFD challenge, was more similar to our previous results in the very long-chain acyl-CoA dehydrogenase-deficient mouse model. ${ }^{14}$

Despite high $C p t-1 b$ expression, we also found, however, that microvesicular fatty change was significantly greater in CPT-1a $+/-$ livers as compared with diet-induced liver steatosis in CPT-1a $+/+$ mice. However, CPT- $1 \mathrm{a}+/-$ mice having greater amounts of microvesicular liver fat may not be inconsistent with them being more insulin-sensitive. The appearance of microvesicular fat is likely a consequence of the FAO deficiency ${ }^{24-26}$ that is unrelated to their heightened insulin sensitivity. ${ }^{27-29}$ This is further supported by a previous study using CPT-1 inhibitor treatment in mice, showing severe microvesicular fatty liver in the absence of insulin resistance. ${ }^{27}$ Furthermore, studies using mice with 
acyl-CoA:diacylglycerol acyltransferase deficiency also demonstrated a dissociation between hepatic steatosis and insulin resistance. ${ }^{30}$

It may also be important to note that differences in glucose and insulin tolerance, as well as fasting insulin levels between the two genotypes and diets may also be explained by different contributions of either hepatic or peripheral sensitivity. As described previously by others, ${ }^{3,22,31}$ in the pre-diabetic state, insulin resistance could occur separately and/or with different degrees in liver versus skeletal muscles, and realized as either impaired fasting glucose or as impaired glucose tolerance.

We measured size of islet by immunostaining $\beta$-cells for insulin. We expected to see either hyperplasia or hypertrophy as a result of excessive secretion from a predicted fatty acid build-up, or alternatively, a $\beta$-cell loss as a result of longterm excess fatty acid exposure. We found that HFD-fed CPT-1a + / - mice had lower percent islet to pancreas area, indicating that they did not have as many insulin-containing $\beta$-cells as did the CPT- $1 \mathrm{a}+/+$ mice. The lower number of $\beta$-cells could have been a result of apoptosis or reduction in the production of insulin granules within the $\beta$-cells. ${ }^{32,33} \mathrm{We}$ did not find degenerative lesions of islets in any of the groups. A fatty acid-induced decreased $\beta$-cell function would explain the decreased glucose tolerance found in HFD-fed CPT-1a $+/-$ mice. In support of these conclusions, a recent study demonstrating that overexpression of SREBP-1c in $\beta$-cells, which resulted in $\beta$-cell triglyceride build-up, caused the onset of impaired glucose tolerance, which these investigators attributed partially to the reduction in number and size of islets. ${ }^{33}$

CPT-1a+/- mice fed the HCD were also found to have lower percent islet area/pancreas area, lower total islet area and lower average islet area. In contrast to CPT-1a $+/-$ mice fed the HFD, these mice were more glucose tolerant and had higher first-phase insulin secretion, indicating that the lower islet area either did not have an effect on their glucose tolerance because of their heightened insulin sensitivity, or that the lower islet area may have been a result of their heightened insulin sensitivity. We interpret the global reduction in islet mass as a response to the overall improved insulin sensitivity as reflected in the CPT- $1 \mathrm{a}+/-$ mice fed either diet.

In conclusion, CPT-1a $+/-$ mice were found to be more insulin-sensitive when fed either a HCD or a HFD, which we speculate may be explained by increased CPT-1b activity in liver. Findings of lower fasting insulin levels in CPT-1a + /mice fed HFD for either 28 or 46 weeks further supported the finding of increased insulin sensitivity from insulin tolerance testing. CPT-1a $+/-$ mice were more glucose tolerant when fed the HCD but less glucose tolerant when fed the HFD. We conclude that this difference was due to an impaired insulin response to glucose as a result of both the HFD and the FAO deficiency. This was further supported by the results indicating that CPT- $1 \mathrm{a}+/-$ mice fed HFD had lower percent islet/pancreas area, lower total islet area, lower number of islets and lower average number of islets per $\mathrm{mm}^{2}$ of pancreas. In contrast, the CPT- $1 \mathrm{a}+1-$ mice fed HCD were also found to have lower percent islet/pancreas area, lower total islet area and lower average number of islets per $\mathrm{mm}^{2}$ of pancreas; however, they were still more glucose tolerant, and this may be attributed to their greater insulin sensitivity.

Thus, the interaction of the CPT-1a deficiency with either HCD or HFD includes increased insulin sensitivity when fed either diet along with enhanced $\beta$-cell responsiveness in those fed the HCD, whereas, the HFD appeared to be detrimental to $\beta$-cell response. There was a decrease in islet cell mass in mutants fed either diet that corresponded with their increased insulin sensitivity. These findings indicate that it is the interaction between genotype and diet that determines metabolic phenotype rather than diet or genotype alone. Therefore, full consideration of the genetic determinants of disease risk should be considered in the context of the individual genotype along with environment and in this case the diet.

\section{Conflict of interest}

The authors declare no conflict of interest.

\section{Acknowledgements}

We are grateful to the late Jane Hosmer and Alvin Almodovar for providing excellent assistance. The project was supported by the following grants: RO1RR02599 (PAW), UL1RR025777 (BAG: UAB-Center of Clinical and Translational Science), P30DK56336 (TRN, BAG, PAW: Nutrition and Obesity Research Center) and P60DK079626 (BAG: UAB-Diabetes Research and Training Center); all are components of the National Institutes of Health, and contents of this work are solely the responsibility of the authors and do not necessarily represent the official views of the National Center for Research Resources, the National Institute for Diabetes, Digestive and Kidney Disease or the NIH.

\section{Author contributions}

LRN: managed and performed a majority of the experiments, analyzed data, lead writer of the manuscript. LT: performed experiments, analyzed data, reviewed the manuscript. DAH: performed experiments, analyzed data, reviewed the manuscript. TRS: performed morphological studies and analyzed data, wrote parts of the manuscript. BAG: performed analyte analyses, reviewed the manuscript. TRN: performed body composition analyses, reviewed the manuscript. PAW: oversaw the entire project, reviewed all data, major role in writing manuscript and obtained primary funding for project. 


\section{References}

1 Costa A, Conget I, Gomis R. Impaired glucose tolerance: is there a case for pharmacologic intervention? Treat Endocrinol 2002; 1: 205-210.

2 Haffner SM, Miettinen H, Gaskill SP, Stern MP. Decreased insulin action and insulin secretion predict the development of impaired glucose tolerance. Diabetologia 1996; 39: 1201-1207.

3 Abdul-Ghani MA, Tripathy D, DeFronzo RA. Contributions of beta-cell dysfunction and insulin resistance to the pathogenesis of impaired glucose tolerance and impaired fasting glucose. Diabetes Care 2006; 29: 1130-1139.

4 Savage DB, Petersen KF, Shulman GI. Disordered lipid metabolism and the pathogenesis of insulin resistance. Physiol Rev 2007; 87: 507-520.

5 Dobbins RL, Szczepaniak LS, Bentley B, Esser V, Myhill J, McGarry JD. Prolonged inhibition of muscle carnitine palmitoyltransferase-1 promotes intramyocellular lipid accumulation and insulin resistance in rats. Diabetes 2001; 50: 123-130.

6 Kashyap S, Belfort R, Gastaldelli A, Pratipanawatr T, Berria R, Pratipanawatr $\mathrm{W}$ et al. A sustained increase in plasma free fatty acids impairs insulin secretion in nondiabetic subjects genetically predisposed to develop type 2 diabetes. Diabetes 2003; 52: 2461-2474.

7 Bollheimer LC, Skelly RH, Chester MW, McGarry JD, Rhodes CJ. Chronic exposure to free fatty acid reduces pancreatic beta cell insulin content by increasing basal insulin secretion that is not compensated for by a corresponding increase in proinsulin biosynthesis translation. J Clin Invest 1998; 101: 1094-1101.

8 Dobbins RL, Chester MW, Daniels MB, McGarry JD, Stein DT. Circulating fatty acids are essential for efficient glucosestimulated insulin secretion after prolonged fasting in humans. Diabetes 1998; 47: 1613-1618.

9 Bjorklund A, Yaney G, McGarry JD, Weir G. Fatty acids and betacell function. Diabetologia 1997; 40: B21-6.

10 McGarry JD, Dobbins RL. Fatty acids, lipotoxicity and insulin secretion. Diabetologia 1999; 42: 128-138.

11 Lee Y, Hirose H, Ohneda M, Johnson JH, McGarry JD, Unger RH. Beta-cell lipotoxicity in the pathogenesis of non-insulindependent diabetes mellitus of obese rats: impairment in adipocyte-beta-cell relationships. Proc Natl Acad Sci USA 1994; 91: 10878-10882.

12 Lehtihet M, Welsh N, Berggren PO, Cook GA, Sjoholm A. Glibenclamide inhibits islet carnitine palmitoyltransferase 1 activity, leading to PKC-dependent insulin exocytosis. Am J Physiol Endocrinol Metab 2003; 285: E438-E446.

13 Zhang D, Liu ZX, Choi CS, Tian L, Kibbey R, Dong J et al. Mitochondrial dysfunction due to long-chain acyl-CoA dehydrogenase deficiency causes hepatic steatosis and hepatic insulin resistance. Proc Natl Acad Sci USA 2007; 104: 17075-17080.

14 Zhang D, Christianson J, Liu Z-X, Tian L, Cheol Soo C, Neschen S et al. Resistance to high-fat diet-induced obesity and insulin resistance in mice with very long-chain acyl-CoA dehydrogenase deficiency. Cell Metab 2010; 11: 1-10.

15 Akkaoui M, Cohen I, Esnous C, Lenoir V, Sournac M, Girard J et al. Modulation of the hepatic malonyl-CoA-carnitine palmitoyltransferase 1A partnership creates a metabolic switch allowing oxidation of de novo fatty acids. Biochem J 2009; 420: 429-438.

16 Nyman LR, Cox KB, Hoppel CL, Kerner J, Barnoski BL, Hamm DA et al. Homozygous carnitine palmitoyltransferase 1a (liver isoform) deficiency is lethal in the mouse. Mol Genet Metab 2005; 86: 179-187.

17 Nagy TR, Clair AL. Precision and accuracy of dual-energy X-ray absorptiometry for determining in vivo body composition of mice. Obes Res 2000; 8: 392-398.

18 Goetzman ES, Tian L, Nagy TR, Gower BA, Schoeb TR, Elgavish A et al. HIV protease inhibitor ritonavir induces lipoatrophy in male mice. AIDS Res Hum Retroviruses 2003; 19: 1141-1150.

19 Kuzuya T, Nakagawa S, Satoh J, Kanazawa Y, Iwamoto Y, Kobayashi $\mathrm{M}$ et al. Report of the Committee on the classification and diagnostic criteria of diabetes mellitus. Diabetes Res Clin Pract 2002; 55: 65-85.

20 Livak KJ, Schmittgen TD. Analysis of relative gene expression data using real-time quantitative PCR and the 2(-delta delta C(T)) method. Methods 2001; 25: 402-408.

21 Stoehr JP, Nadler ST, Schueler KL, Rabaglia ME, Yandell BS, Metz SA et al. Genetic obesity unmasks nonlinear interactions between murine type 2 diabetes susceptibility loci. Diabetes 2000; 49: 1946-1954.

22 Bonnefont JP, Demaugre F, Prip-Buus C, Saudubray JM, Brivet M, Abadi $\mathrm{N}$ et al. Carnitine palmitoyltransferase deficiencies. Mol Genet Metab 1999; 68: 424-440.

23 Bruce CR, Hoy AJ, Turner N, Watt MJ, Allen TL, Carpenter K et al. Overexpression of carnitine palmitoyltransferase- 1 in skeletal muscle is sufficient to enhance fatty acid oxidation and improve high-fat diet-induced insulin resistance. Diabetes 2009; 58: 550-558.

24 Letteron P, Fromenty B, Terris B, Degott C, Pessayre D. Acute and chronic hepatic steatosis lead to in vivo lipid peroxidation in mice. J Hepatol 1996; 24: 200-208.

25 Fromenty B, Pessayre D. Inhibition of mitochondrial beta-oxidation as a mechanism of hepatotoxicity. Pharmacol Ther 1995; 67: 101-154.

26 Fraser JL, Antonioli DA, Chopra S, Wang HH. Prevalence and nonspecificity of microvesicular fatty change in the liver. Mod Pathol 1995; 8: 65-70.

27 Grefhorst A, Hoekstra J, Derks TG, Ouwens DM, Baller JF, Havinga $\mathrm{R}$ et al. Acute hepatic steatosis in mice by blocking beta-oxidation does not reduce insulin sensitivity of very-lowdensity lipoprotein production. Am J Physiol Gastrointest Liver Physiol 2005; 289: G592-G598.

28 Deschamps D, Fisch C, Fromenty B, Berson A, Degott C, Pessayre D. Inhibition by salicylic acid of the activation and thus oxidation of long chain fatty acids. Possible role in the development of Reye's syndrome. J Pharmacol Exp Ther 1991; 259: 894-904.

29 Kim JK, Kim YJ, Fillmore JJ, Chen Y, Moore I, Lee J et al. Prevention of fat-induced insulin resistance by salicylate. J Clin Invest 2001; 108: 437-446.

30 Monetti M, Levin MC, Watt MJ, Sajan MP, Marmor S, Hubbard BK et al. Dissociation of hepatic steatosis and insulin resistance in mice overexpressing DGAT in the liver. Cell Metab 2007; 6: 69-78.

31 Faerch K, Brons C, Alibegovic AC, Vaag A. The disposition index: adjustment for peripheral vs hepatic insulin sensitivity? J Physiol 2010; 588: 759-764.

32 Bollheimer LC, Kemptner DM, Kagerbauer SM, Kestler TM, Wrede CE, Buettner R. Intracellular depletion of insulin: a comparative study with palmitate, oleate and elaidate in INS-1 cells. Eur J Endocrinol 2003; 148: 481-486.

33 Takahashi A, Motomura K, Kato T, Yoshikawa T, Nakagawa Y, Yahagi $\mathrm{N}$ et al. Transgenic mice overexpressing nuclear SREBP-1c in pancreatic beta-cells. Diabetes 2005; 54: 492-499.

(c)


Derivative Works 3.0 Unported License. To view a copy of this license, visit http://creativecommons.org/ licenses/by-nc-nd/3.0/ 